Meta

Journal des traducteurs

Translators' Journal

\title{
Défense et illustration de l'approche herméneutique en traduction
}

\section{Ioana Balacescu et Bernd Stefanink}

Volume 50, numéro 2, avril 2005

Processus et cheminements en traduction et interprétation Processes and Pathways in Translation and Interpretation

URI : https://id.erudit.org/iderudit/011007ar

DOI : https://doi.org/10.7202/011007ar

Aller au sommaire du numéro

\section{Éditeur(s)}

Les Presses de l'Université de Montréal

\section{ISSN}

0026-0452 (imprimé)

1492-1421 (numérique)

Découvrir la revue

\section{Citer cet article}

Balacescu, I. \& Stefanink, B. (2005). Défense et illustration de l'approche herméneutique en traduction. Meta, 50(2), 634-642.

https://doi.org/10.7202/011007ar

\section{Résumé de l'article}

Depuis un demi-siècle, l'approche herméneutique a souffert de la dominance des approches analytiques en traduction, victimes du « mythe de

l'objectivisme " et de l'engouement pour une illusoire traduction automatique. Elle connaît un regain d'intérêt depuis la remise en question de ces approches analytiques, qui se sont soldées par un échec relatif de la traduction automatique. Ce regain d'intérêt est d'autant plus puissant que les descriptions heuristiques de l'activité traduisante fournies par les herméneutes se voient légitimées scientifiquement par les découvertes des chercheurs cognitivistes ainsi que par les auto-témoignages des praticiens de la traduction. Les recherches cognitivistes viennent ainsi alimenter le nouveau critère d'évaluation de la « intersubjektive Nachvollziehbarkeit » réclamé par Stefanink, dès 1997, à la suite du bouleversement épistémologique en traductologie. 


\title{
Défense et illustration de l'approche herméneutique en traduction
}

\author{
IOANA BALACESCU \\ Université de Craiova, Craiova, Roumanie \\ ioanadi@hotmail.com
}

BERND STEFANINK

Université de Bielefeld, Bielefeld, Allemagne bstefanink@hotmail.com

\begin{abstract}
RÉSUMÉ
Depuis un demi-siècle, l'approche herméneutique a souffert de la dominance des approches analytiques en traduction, victimes du «mythe de l'objectivisme» et de l'engouement pour une illusoire traduction automatique. Elle connaît un regain d'intérêt depuis la remise en question de ces approches analytiques, qui se sont soldées par un échec relatif de la traduction automatique. Ce regain d'intérêt est d'autant plus puissant que les descriptions heuristiques de l'activité traduisante fournies par les herméneutes se voient légitimées scientifiquement par les découvertes des chercheurs cognitivistes ainsi que par les auto-témoignages des praticiens de la traduction. Les recherches cognitivistes viennent ainsi alimenter le nouveau critère d'évaluation de la «intersubjektive Nachvollziehbarkeit » réclamé par Stefanink, dès 1997, à la suite du bouleversement épistémologique en traductologie.
\end{abstract}

\begin{abstract}
During the last fifty years translation studies have been dominated by analytical approaches, victims of the "myth of objectivism" and an illusory belief in machine translation. The hermeneutic tradition lived an underground life. The relative failure of machine translation has raised questions about the validity of analytical approaches. But what furthered a new interest in an hermeneutic approach to translations studies most were the results of cognitive research which furnished a scientific legitimating to the descriptions from the heuristic perspective of translational activities, the more so as these descriptions are confirmed by the testimony of practitioners in translation. Thus cognitivistic research provides the basis for the new evaluation criterion of "intersubjektive Nachvollziehbarkeit" (intersubjective understanding) exacted by the epistemological revolution as demanded by Stefanink (1997).
\end{abstract}

\section{MOTS-CLÉS/KEYWORDS}

herméneutique, cognitivisme, bouleversement épistémologique en traduction, «intersubjektive Nachvollziehbarkeit », formation du traducteur.

\section{Défense et illustration de l'approche herméneutique en traduction}

L'approche herméneutique en traduction n'a pas l'attention qu'elle mérite. D’une part, parce qu'elle est associée à l'exégèse partiale des textes bibliques telle qu'elle a été pratiquée dans la tradition ecclésiastique, et, d'autre part, parce que ses bases philosophiques quelque peu hermétiques lui ont valu une réputation de mysticisme «pré-scientifique». 
Et ceci à tort! Car le traducteur qui sait s'ouvrir au discours herméneutique sans préjugés - ce qui est justement un des principes fondamentaux de la lecture des textes, telle qu'elle est préconisée par l'herméneutique - comprendra vite que si cette approche théorique ne lui fournit certes pas un ensemble de règles clairement définies pour arriver à une traduction dite «objective» (à laquelle prétendent un certain nombre d'approches, comme celle que nous recommandent, par exemple GerzymischArbogast et Mudersbach 1998, qui se déclarent les seules à pouvoir accéder au statut d'une «science»), elle est par contre seule à bâtir une théorie qui, bien que partant d'une réflexion théorique, semble bel et bien décrire la pratique quotidienne du traducteur. Par contre, les procédures d'analyse élaborées par Gerzymisch-Arbogast et Mudersbach, dans le cadre du site protégé de la tour d'ivoire universitaire, ne tiennent nullement compte de la déchirure du traducteur, qui relève peut-être moins de l'opposition sourcier-cibliste (les deux âmes faustiennes que tout traducteur porte en lui) que de sa soumission aux contraintes de «fidélité» au texte source (TS), et à celle de son «être en situation».

\section{L'illusion de l'objectivité en traduction}

En effet, trop longtemps les traductologues se sont bercés dans l'illusion de pouvoir arriver à une traduction «objective». Sous l'influence du structuralisme, les théoriciens, qui ont considéré le mot comme unité de traduction, ont pensé atteindre cette objectivité par une analyse en sèmes des mots du TS afin de trouver en LC un ou plusieurs mots reproduisant ces sèmes. Après l'échec de ces recherches (de la machine à traduire), ces mêmes théoriciens ont étendu leur champ de recherches à la phrase, espérant aboutir à l'objectivité en réduisant l'activité traduisante à un certain nombre de «transpositions» en inventaire clos. C'était l'époque de la stylistique comparée. Avec la linguistique du texte, on a finalement compris qu'on ne pouvait pas traduire en cherchant des équivalences au niveau des micro-structures. Le texte entier était devenu l'unité de traduction ${ }^{1}$. On avait compris qu'il fallait traduire le sens du texte et que celui-ci n'était pas simplement égal à la somme des significations des mots qui le constituent. Qui plus est, on avait découvert qu'il y avait des «lectures plurielles» du texte (R. Barthes) $)^{2}$ et que celui-ci était une « œuvre ouverte» (U. Eco). Cela n'a pourtant pas découragé certains théoriciens, comme Gerzymisch-Arbogast / Mudersbach (1998) de persister dans la recherche de l'objectivité, en soumettant le texte à un certain nombre de démarches analytiques - toutes préalables à l'acte traduisant proprement dit, qui devaient permettre l'accès au sens.

\section{La «spirale herméneutique» : processus «bottom up » et «top down »}

Les herméneutes dénoncent ces illusions concernant l'accès au sens «objectif» du texte: "L'accès au sens ne se fait pas par le biais de l'analyse» (Stolze 2003: 162). En effet, se basant sur des réflexions de philosophes herméneutes, comme Heidegger ou Gadamer, les traductologues herméneutes savent que le sens n'est pas dans le texte, une fois pour toutes, mais qu'il se construit dans un va-et-vient dialectique entre le texte et le récepteur. Le récepteur ne peut comprendre le texte qu'en fonction de son vécu, de son world knowledge. C'est ce qu'on a appelé le «cercle herméneutique » qui, traditionnellement, a toujours été considéré comme un cercle "vicieux». 
Heidegger a innové face à cette tradition herméneutique, alléguant que ce cercle n'avait rien de vicieux mais, au contraire, offrait, la chance de pénétrer plus profondément la vérité du texte:

Le «cercle herméneutique» ne doit pas être rabaissé au rang d'un vitiosum fût-il toléré. Il recèle un potentiel positif d'accès à la connaissance la plus profonde qui, toutefois ne pourra être saisi réellement qu'au moment où l'interprétation aura compris que sa tâche première, permanente et ultime sera de ne pas laisser préfigurer son projet, sa prévision et son action par des idées reçues, mais de s'assurer du thème scientifique à partir de la chose elle-même (Heidegger 1927/1993: 312; notre traduction).

La deuxième partie de cette citation nous révèle une des conditions fondamentales pour l'accès au sens: le récepteur doit être conscient de sa «facticité » (pour parler en termes sartriens), c'est-à-dire du fait qu'il est toujours «en situation » et que sa vision du texte sera toujours conditionnée par son vécu personnel. Il doit faire le grand écart entre, d'une part, son vécu en tant que fondement de sa saisie du sens et, d'autre part, son vécu en tant que déformateur de sa compréhension du texte.

Ainsi compris, le cercle herméneutique devient une "spirale herméneutique» assurant une compréhension de plus en plus adéquate au fur et à mesure que le récepteur progresse dans la lecture du texte, les mots étant le stimulus qui active chez le récepteur certaines voies neuronales, créées par des expériences similaires enregistrées dans sa mémoire. C'est la similitude entre les expériences déjà vécues et l'information potentielle mise à disposition dans les mots du texte qui permet la compréhension du texte en raison du processus de catégorisation général sous-jacent à toute compréhension humaine, comme nous l'apprennent les cognitivistes (Lakoff 1988). Ainsi, au fur et à mesure que le récepteur progresse dans la lecture du texte, un choix de plus en plus restreint et précis s'opère au niveau des sphères neuronales activées en fonction des isotopies du texte qui se dégagent avec une précision de plus en plus grande.

Les découvertes des cognitivistes viennent ainsi confirmer les hypothèses des herméneutes. Là où Gadamer parlait d'un «dialogue avec le texte», les cognitivistes parlent, plus prosaïquement, de processus bottom-up et top-down. La grande différence entre ces deux courants de pensée se situe au niveau de l'attitude du récepteur. Pour les cognitivistes, celui-ci participe activement à l'acte de compréhension, il «gère» le langage (cf. le terme de «Sprachverarbeitung» utilisé par ex. dans Rickheit et Strohner 1993). Pour les herméneutes, il est passif: la vérité du texte s'impose irrésistiblement à lui, à condition qu'il arrive à gérer consciemment les scripts, schémas (pour utiliser la terminologie des cognitivistes) ou plus simplement des attentes avec lesquels il va aborder le texte et qui peuvent devenir un obstacle à son ouverture à la vérité du texte, s'il n'en prend pas conscience.

\section{La «fusion des horizons» chez le traducteur herméneute}

Reprenant la conception gadamerienne de l'acte de compréhension comme un dialogue avec le texte, qui aboutit finalement à une «fusion des horizons» («Horizontverschmelzung») dans laquelle le personnage du récepteur et la vérité du texte ne font plus qu'un, les traductologues herméneutes ne conçoivent plus le traducteur comme un intermédiaire "entre» le TS et le TC, mais comme un herméneute dans la conscience duquel le TS et le TC fusionnent. Le traducteur n'est donc plus un «passeur» 
qui fait passer le sens du TS au TC $\mathrm{TC}^{3}$. Finie aussi la recherche des «équivalences» au niveau des micro-structures. Le passage du TS au TC se fait de façon intuitive: le traducteur/récepteur du TS est «saisi» par la vérité du TS, qui s’impose à lui avec

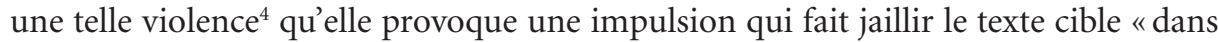
un processus mental en partie inconscient» (Stolze 2003: 211), faisant passer le sens de l'« enveloppe» de la LS dans celle de la LC.

Écoutons un praticien de la traduction poétique que l'on ne soupçonnera certainement pas d'avoir écrit ces phrases pour les besoins de la cause, c'est-à-dire pour prouver un point de vue théorique, tel qu'il est exposé par la traductologue Stolze, puisqu'il commence son article en déclarant fièrement: "Io non possiedo teorie et tanto meno recette sul tradurre» (Coco 2003 : 132) et cite Newmark à l'appui: "Non esiste, né mai esisterà, una scienza della traduzione (Coco 2003: 133). Et pourtant il est, comme M. Jourdain, inconsciemment tributaire de l'approche herméneutique, ce qui semble plaider pour le caractère naturel et pratique de cette approche:

O sono un misto di spagnolo e di italiano. È un gioco infinito! Alla fine finisco per confodermi io

Stesso perché non ricordo più se si tratta di una traduzione dall'italiano in spangolo o viceversa.

O se tale traduzione ci sia mai stata! (Coco 2003: 147)

La «fusion des horizons» postulée par la théorie de Gadamer n'est-elle pas démontrée à la perfection dans la pratique de ce traducteur?

La sémantique fillmorienne des «scenes-and-frames» nous aide à comprendre ce processus. Fillmore (1976) considère les mots comme des «linguistic frames» qui font naître en nous des «cognitive scenes». Au traducteur de trouver les «frames» linguistiques dans lesquels il pourra couler les «scenes» présentes dans son esprit. Pour les herméneutes, il ne s'agit même pas d'un effort conscient de la part du traducteur, mais d'une pression exercée par la vérité du TS qui impose impérativement les mots du TC. Heidegger nous fournit les fondements philosophiques de ce processus dans son ontologie: "Ce sont les mots qui viennent aux significations et non les significations qui viennent aux mots.»

\section{«Vouloir dire de l'auteur" vs caractère inachevé de la traduction et plausibilité intersubjective}

Le fait que le sens ainsi traduit est toujours fonction du vécu personnel du récepteur/ traducteur a pour conséquence qu'il est illusoire de croire pouvoir atteindre à l'objectivité en traduction, voire de retrouver le "vouloir-dire de l'auteur», (comme le préconise par ex. l'École de Paris; cf. Lederer 1994). Le traducteur doit traduire «ce qu'il comprend» (Stolze 2003: 155), c'est-à-dire «ce qui est mentalement présent chez lui » (id. ibid. 248). Il ne jouit cependant pas d'une liberté incontrôlée, telle que la prônent par exemple les représentants de la «Manipulation School», qui affirment que, de toute façon, chaque traduction est déjà une «manipulation » du TS, rendant vain tout espoir d'accéder à une traduction objective et réduisant finalement les critères d'évaluation à la seule affirmation du traducteur que sa traduction est bien une traduction, quelle que soit l'importance des «manipulations » auxquelles il a soumis le TS. 
Le traducteur herméneute, quant à lui, se doit de soumettre son premier jet intuitif en LC à une évaluation, susceptible de légitimer sa traduction. Cette évaluation doit être plausible pour autrui, la "plausibilité intersubjective» venant remplacer l'objectivité comme critère d'évaluation (cf. le concept de «intersubjektive Nachvollziehbarkeit» introduit par Stefanink, 1997). La plausibilité intersubjective doit être assurée par l'observation d'un certain nombre de règles conformes à la conception herméneutique d'une linguistique du texte telle que la conçoit Eugenio Coseriu:

Ceci veut dire que le contenu une fois compris doit être mis en rapport avec un élément textuel précis, qu'on doit montrer qu'au signifié du macro-signe dans le texte correspond une certaine expression. De ce point de vue la linguistique du texte, telle qu'elle est comprise ici, est interprétation, est herméneutique. (Coseriu 1980: 151, notre traduction)

«Autrui» est dans ce cas l'expert en traduction (Risku 1998: 88ss.), celui-ci pouvant être l'auteur lui-même. Dans ce dernier cas le processus d'évaluation peut devenir un automatisme et se manifester dans les modifications apportées directement au TC (Stolze 2003: 240). Ces modifications peuvent se continuer à l'infini, car au gré de son empathie croissante avec le texte, le récepteur participe aussi de plus près à la vérité du texte. Le texte comporte, en effet, toujours un "potentiel de surplus de sens» («Sinnüberschuss» Stolze 2003: 73) qui fait que chaque lecture - y compris celle de l'auteur lui-même - produit à chaque fois un nouveau sens. Ce que la théoricienne roumaine de la poïétique qu'est Irina Mavrodin, a rendu par la triade poïétique - poétique - "poïétique», la poétique étant l'acte créateur, la poétique l'œuvre créée et la "poïétique» le nouvel acte créateur par le récepteur du texte créé. Ce processus de compréhension n'est jamais achevé; Stolze (2003: 222) parle de la «Unabschließbarkeit des tentativen Entwurfs». Les bases philosophiques de ce processus nous sont fournies par l'ontologie heidegerienne qui dit que la compréhension n'est plus une méthode pour s'approprier un sens étranger, mais que comprendre c'est créer un sens (Sinnstiftung), idée qui est reprise par Gadamer:

Ce n'est pas occasionnellement, mais toujours que le sens d'un texte dépasse son auteur. C'est pour cela que comprendre n'est pas seulement un comportement reproductif, mais toujours aussi un comportement productif (Gadamer 1960: 280; notre traduction).

\section{L'aspect ludique de l'activité traduisante}

La compréhension du texte autant que la production du texte se font sur le mode du jeu. Il est frappant de constater à quel point cette idée du sens qui se crée par jeu se retrouve dans diverses approches théoriques récentes. La praticienne et théoricienne de la traduction qu'est Irina Mavrodin, revient toujours sur l'aspect ludique de sa pratique traduisante. Ainsi, après avoir relaté les frustrations auxquelles s'expose le traducteur dans sa relation avec la maison d'édition, Mavrodin (2001: 118) évoque le « contrepoids rédempteur» de la «dimension ludique», le «jeu avec les mots », allant même jusqu'à constater que son activité de traducteur a développé son côté ludique, et de par là sa créativité, sans laquelle la traduction est impossible:

Dans tout ce - disons - métier, de la langue, de la parole, la créativité sine qua non dont nous avons parlé, mon côté ludique s'est développé à travers la traduction: il n'a pas été annihilé, tout au contraire. Quand je choisis un mot, j'ai la sensation que je me jette dans un abîme. Je joue ou je risque ou j'ai le goût de ce mot. (Mavrodin 2001: 119) 
De même, Fritz Paepcke, traductologue et fondateur de l'École de Heidelberg, compare la recherche de la «solution heureuse» d'un problème de traduction à un jeu de football au cours duquel les joueurs se font des passes devant les buts adverses, jusqu'à ce que l'un d'eux, à la suite d'une passe au centre bien placée, trouve l'ouverture dans la défense adverse pour marquer le but. L'importance que Paepcke attache à l'aspect ludique que comporte pour lui l'activité traduisante se reflète jusque dans le titre de son traité de traductologie, Ouvertures sur la traduction (Paepcke 1981), où ouvertures - en dehors du sens contextuel dans le titre - fait allusion à la scène du jeu de football évoquée ci-dessus. Quelques années plus tard, il formulera catégoriquement: "Le mode sur lequel sont fabriqués les textes et leur traduction est celui du jeu» (Paepcke 1986: 126; notre traduction). Là encore, la paternité de ces idées revient aux philosophes: ainsi pour Wittgenstein comprendre est possible grâce à la participation au Sprachspiel (= jeu de langage), conception qui est reprise par Gadamer dans Wahrheit und Methode (1960: 101 ss.).

Les chercheurs en créativité sont venus confirmer ses hypothèses, en apportant la confirmation jusque dans le titre allemand du livre sur la créativité de Bono, intitulé Lateral Thinking, qui a été traduit par Spielerisches Denken (littéralement: la pensée ludique). Quant au traductologue, Kußmaul (2000) a apporté la preuve empirique de l'importance de cet esprit ludique au moyen de ses analyses conversationnelles de traducteurs confrontés à un problème de traduction: la solution à ce problème venait souvent au cours d'une activité parallèle de type ludique (retournement de la cassette enregistreuse du débat, préparation d'une tasse de café, etc.), alors que la pensée «convergente» pour parler avec le chercheur en créativité Guilford (1971) (de Bono parle de pensée «horizontale») est abandonnée au profit d'une pensée « divergente»/ «latérale» qui «joue» à envisager le problème sous d'autres perspectives. À la différence des philosophes herméneutes toutefois, qui insistent sur le caractère naturel et spontané de ce jeu, les chercheurs en créativités, cognitivistes instrumentalisent ce jeu pour en faire un outil solutionneur de problèmes.

Le témoignage donné par le praticien, traducteur de poésie, Emilio Gioco de ce que les chercheurs en créativité appelleraient la phase d'incubation, vient confirmer ces observations de Kußmaul:

Io gioco con le parole, così come il bambino gioca con i giocattoli. E spesso mi sorprendo, nei vari momenti della giornata (mentre mangio, mentre aiuto mia moglie nei lavori domestici, persino quando sto riposando), a contare sillabe con le dita, tamburellando sul tavolo, sul bracciolo della poltrona a cui sto togliendo la polvere, o sulle mie cosce, sul petto, sulla faccia. (Gioco 2003: 137)

\section{Un jeu avec les souvenirs}

Après avoir accepté l'idée wittgensteinienne du Sprachspiel et avoir compris à quoi on joue (à savoir à découvrir la vérité du texte), il faut se demander avec quoi on joue. Et là la réflexion cognitiviste nous est d'un grand secours. Elle nous apprend, en effet, que nous ne comprenons qu'en fonction de ce qu'a enregistré notre mémoire. Des chercheurs en mémoire, comme Schank (1982), nous ont appris que nos expériences vécues - nos souvenirs en quelque sorte - sont stockées, entre autres, sous forme de MOPs (Memory Organisation Packages) c'est-à-dire sous forme d'éléments scéniques vécus faisant partie de scenes plus vastes, et reliés les uns aux autres par des voies 
neuronales, plus ou moins bien développées selon la fréquence des expériences vécues en question. Ces voies neuronales sont activées par les stimuli - les frames linguistiques au sens fillmorien du terme - que sont les mots du texte, lesquels appellent dans la mémoire active les éléments scéniques stockés dans la mémoire longue ${ }^{5}$, nos souvenirs.

\section{Quelles conséquences pour la formation des traducteurs?}

Dans une de ces interviews, Irina Mavrodin (2001: 123) dit «qu'il serait dommage de partir sans transmettre les différents petits secrets, trucs, artifices dont [elle] a eu grand besoin quand [elle] a commencé à traduire». Tirer des leçons de sa pratique, en dégager une réflexion théorique et vouloir en déduire un enseignement est, en effet, une démarche assez naturelle de tout expert dans un domaine. Que nous offrent donc les herméneutes en matière de didactique de la traduction?

À première vue et par rapport à d'autres théories, l'hypothèse herméneutique ne nous fournit pas un «kit» didactique très concret, comparable à l'outillage mis à disposition par la linguistique structuraliste, la linguistique pragmatique ou la linguistique du texte. Dans l'optique structuraliste, la tâche du didacticien de la traduction était en effet clairement formulée: il suffisait d'apprendre à analyser en traits distinctifs les mots du TS et trouver un ou plusieurs mots susceptibles de les rendre en LC. Eugene Nida ne s'était-il pas permis d'affirmer que "What we do aim at is a faithful reproduction of the bundles of componential features» (1974: 50) ${ }^{6}$. De même, à un niveau supérieur, la pragmatique nous apprenait d'une part l'existence des actes de langage et de la prise en considération de la situation de communication, ce qui permettait, une fois la situation de communication analysée, de trouver en LC des équivalents culturels légitimés objectivement par des analyses contrastives (cf. Hönig et Kußmaul 1982). D’autre part, la stylistique comparée qui, elle aussi, avait pris la phrase comme unité de traduction nous apprenait qu'il suffisait de prendre conscience de la notion de «transposition» et d'adapter les différents types de transposition - mis en évidence par Vinay/Darbelnet (1958) à un niveau général - aux langues en présence comme l'ont fait Bausch (1968), par exemple, pour l'allemand et Cristea (1998) pour le roumain. Les linguistes du texte finalement, dans leur manifestation extrême, comme nous la trouvons chez Gerzymisch-Arbogast et Mudersbach (1998) ont eu la prétention de dépasser toutes ces approches micro-structurelles pour enseigner une démarche «scientifique» censée mener à une traduction objective. Cette démarche consistait en une analyse exhaustive du TS sous différents aspects. Cette analyse devait se faire sur fond d'un réseau associatif déclenché par les éléments centraux du TS. De l'analyse contrastive entre ce réseau et le réseau virtuel correspondant en LC devaient se dégager les transpositions culturelles à opérer. Cette «analyse du texte pertinente pour le traducteur» devait impérativement avoir lieu avant toute tentative même partielle de traduction ${ }^{7}$.

L'erreur de toutes ces démarches est le manque de prise en considération de la personne du traducteur. Celui-ci a un vécu personnel qui, comme nous l'avons vu, intervient pour beaucoup dans sa saisie du sens (à traduire). Aussi, dans l'approche herméneutique, l'impératif didactique qui prime sur tout est de faire prendre conscience de cette implication de la personne du traducteur et de sa facticité qui influence sa réception du texte. Un deuxième élément important dans la formation 
du traducteur herméneute, c'est l'élargissement de sa base associative par des lectures de toutes sortes, auxquelles nous invite Stolze (2003 : 307), en quoi elle est largement soutenue par les cognitivistes qui plaident fortement en faveur d'une culture générale, acquise par la lecture (Risku 1998: 163). L'autre façon de se munir d'une base associative large nécessaire à la compréhension du texte est la recherche systématique en vue d'un approfondissement des connaissances concernant le thème du texte à traduire, ce qui sera notamment le cas des textes en langue de spécialité. Enseigner les méthodes de recherche destinées à élargir cette base associative spécifique en rapport avec la spécificité du texte à traduire sera le troisième objectif didactique préconisé dans l'approche herméneutique.

On le voit, on est loin des "petits secrets, trucs, artifices», cités en début de ce chapitre. Faut-il pour autant les négliger? Non, le traducteur doit faire feu de tout bois, et c'est l'ensemble de tous ses apports - la prise de conscience de la structure sémique des mots, de la notion de situation et des «actes de langage » y afférant, des transpositions de la stylistique comparée, de l'analyse textuelle de la linguistique du texte, ainsi que des bases philosophiques et cognitivistes qui conditionnent l'individu traduisant - qui lui permettra de livrer une traduction satisfaisante du point de vue du contenu comme de la forme.

\section{NOTES}

1. Pour le détail de cette vision historique de la traductologie cf. Balacescu et Stefanink 2001 et 2002.

2. Cf. aussi Mavrodin (2001: 110): «La traduction est une herméneutique. [...] Pour le traducteurherméneute, la traduction est une lecture, une parmi d'autres lectures possibles, tu t'inscris dans une isotopie, qui peut être celle-ci, mais qui peut aussi être une autre. »

3. Cf. le titre des mélanges offerts à K. Reiß pour son $70^{\mathrm{e}}$ anniversaire: Traducere Navem, où le sens est considéré comme un navire qu'on fait passer d'une rive à l'autre, d'une langue à l'autre.

4. $\quad$ N.B. : Stolze (2003) utilise le terme de Überwältigtsein du traducteur par la vérité du texte. Ce mot qui selon les contextes peut signifier "être vaincu», "être subjugé» a la même racine que Vergewaltigung qui veut dire «viol», et on pourrait dire que le traducteur est en quelque sorte violé par la vérité du TS, à l'inverse de l'image du viol utilisée par Jean-René Ladmiral (1993) pour qui c'est le traducteur qui viole le texte source. Même idée du viol du texte par le traducteur dans le «Hermeneutic thrust» de George Steiner (1975).

5. Pour une présentation plus détaillée, voir Balacescu et Stefanink 2003.

6. Onze ans plus tard, Nida se distanciera de cette vision de la traduction, centrée sur le mot: «We are no longer limited to the idea that meaning is centered in words or even in grammatical distinctions. Everything in language, from sound symbolism to complex rhetorical structures, carries meaning » (Nida: 1985: 119)

7. Pour plus de détails, voir le c.-r. de Stefanink (1998).

\section{RÉFÉRENCES}

Balacescu, Ioana et B. Stefanink (2001): "Une traductologie au service de la didactique: l'école allemande au sein de la famille traductologique, $1^{\text {ère }}$ partie », Le langage et l'homme. Traductologie - Textologie 36-, p. 89-104.

BAlacescu, Ioana et B. Stefanink (2002) : «Une traductologie au service de ladidactique: l'école allemande au sein de la famille traductologique, $2^{\mathrm{e}}$ partie», Le langage et l'homme. Traductologie - Textologie, 37-1, p. 155-176.

BALACESCU, Ioana et B. STEFANINK (2003): «Du structuralisme au cognitivisme: la créativité au fil des théories de la traduction ", Le langage et l'homme. Traductologie - Textologie, Sciences du langage 38-1 p. 125-145.

Bausch, K-R. (1968): «Die Transposition. Versuch einer neuen Klassifikation», Linguistica Antverpiensia II/1968, p. 29-50. 
Bono, E. de (1970): Lateral Thinking. A Textbook of Creativity.London, Ward Lock Educational. Coco, E. (2003) : «Alcune precisazioni e riflessioni», dans Thiers (éd.), 2003, p. 130-153.

Coseriu, E. (1980): Textlinguistik. Eine Einführung. Hrsg. und bearb. v. J. Albrecht, Tübingen, Narr.

Cristea, T. (1998): Stratégies de la traduction, Bucuresti, Editura Fundatiei «România de mâine».

Fillmore, C. J. (1976): «Scenes-and-Frames Semantics», in Linguistic Structures Processing. (dir.). Antonio Zampolli, Amsterdam: N. Holland, p. 55-88.

Gadamer, H.-G. (1960): Wahrheit und Methode. Grundzüge einer philosophischen Hermeneutik 5. Aufl. 1986, Tübingen: J.C.B. Mohr (Paul Siebeck).

Gerzymisch-Arbogast, H. and Mudersbach, Klaus (1998): Methoden des wissenschaftlichen Übersetzens, Tübingen: Francke (UTB 1990).

Guilford, J. P. (1971): The Nature of Human Intelligence, London: McGraw-Hill.

Heidegger, Martin $\left(1927 / 1993^{17}\right)$ : Sein und Zeit. Tübingen: Niemeyer.

Hönig, H. and Kussmaul, P. (1982): Strategie der Übersetzung. Tübingen: Narr.

Kussmaul, P. (2000): Kreatives Übersetzen. Tübingen, Stauffenburg.

Ladmiral, J.-R. (1993): "Sourciers et ciblistes», Holz-Mänttäri / C. Nord: Traducere Navem. Festschrift für Katharina Reiß. Tampere: studia translatologica, ser. A vol 3.

Lakoff, G. (1987): Women, Fire and Dangerous Things. What Categories Reveal about the Mind. Chicago, University of Chicago Press.

Lederer, M. (1994): La traduction aujourd'hui, Paris, Hachette.

Mavrodin, I. (2001): Cvadratura cercului, Bucuresti, Editura Eminescu.

Mednick, S. A. (1962): «The Associative Basis of the Creative Process», Psychological Review, 69, p. $220-232$.

NidA, E. (1974) : «Semantic Structure and Translating», p. 50, W.Wilss/G.Thome: Aspekte der theoretischen sprachbezogenen und angewandten Uebersetzungswissenschaft II, Heidelberg: Groos.

NidA, E. (1985) : «Translating Means Translating Meaning - A Sociosemiotic Approach to Translating», Bühler.

Paepcke, F. and Forget, P. (1981): Textverstehen und Uebersetzen. Ouvertures sur la Traduction. Heidelberg: Groos.

Paepcke, F. (1986): Im Übersetzen leben. Übersetzen und Textvergleich. Hrsg. von Klaus Berger und Hans-Michael Speier, Heidelberg Winter.

Rickheit, G.and Strohner, H.(1993): Grundlagen der kognitiven Sprachverarbeitung. Modelle, Methoden, Ergebnis, Tübingen: Francke.

Risku, H. (1998): Translatorische Kompetenz: kognitive Grundlagen des Übersetzens als Expertentätigkeit, Tübingen, Stauffenburg.

Schank, R. C. (1982): Dynamic Memory. A Theory of Reminding and Learning in Computers and People, London/New York, Cambridge University Press.

Stefanink, B. (1996): c.-r. de Heidrun Gerzymisch-Arbogast.

Stefanink, B. (1997): " "Esprit de finesse" - "Esprit de géométrie": Das Verhältnis von "Intuition" und "übersetzerrelevanter Textanalyse" beim Übersetzen", dans Rudi Keller (éd.): Linguistik und Literaturübersetzen. Tübingen, Narr 1997, p. 161-184.

Stefanink, B. (1998): c.-r. de Gerzymisch-Arbogast and Mudersbach, FluL, 27, p. 245-248.

Steiner, G. (1975): After Babel: Aspects of Language and Translation, London, Oxford and New York, Oxford University Press.

Stolze, Radegundis (2003): Hermeneutik und Translation, Tübingen, Narr.

Thiers, G. (éd.) (2003): Baratti. Commentaires et réflexions sur la traduction de la poésie, coll. «Isule Literarie. Des îles littéraires", Albiana - Bu - Ccu - Iitm.

Vinay, J.-P. et Darbelnet, J. (1958): Stylistique comparée du français et de l'anglais. Méthode de traduction, Paris, Didier. 